\title{
Determination of distribution of heat-conducting material concentration in protective layer of TPS panel
}

\author{
Lukasz Brodzik ${ }^{1, *}$, Andrzej Frąckowiak ${ }^{1}$ \\ ${ }^{1}$ Poznań University of Technology, Chair of Thermal Engineering, Piotrowo 3 street, 60-965 Poznań, Poland
}

\begin{abstract}
Paper presents the problem of heating the damaged insulation of an orbiter. Changes of the insulation's thermal properties, made by adding conductive material of high value of specific heat in a form of a dope to the protective layer, were examined. The analysis was made using insulating material LI900 and beryllium dope. In the paper was investigated the effect of the dopant's share on the temperature distribution on the aluminum surface. This influence was depended on the size of the damage, defined by parameter $\mathrm{D}$, as well as a constant percentage volume fraction.
\end{abstract}

\author{
Nomenclature \\ $T$ - temperature, \\ $\rho-$ density, \\ $p$ - pressure, \\ $c$ - specific heat, \\ $k$ - thermal conductivity, \\ $\gamma$ - concentration of the addition
}

\section{Introduction}

The object of the research described in this paper is high temperature reusable surface insulation (HRSI) of an orbiter. In extreme cases, the damage of the insulation material results in melting of the orbiter aluminum structure, leading directly to its destruction. Under normal conditions, when the thermal protection system is fully operative, a significant increase in the temperature of the orbiter structure takes place only after the vehicle has landed. When a malfunction occurs, the temperature increase takes place earlier, already at the flight stage. Contrary to many concepts discussed in literature, the paper does not focus on increasing of the thermal durability. The LI900 surface insulation is a wellexplored material $[1,2,3]$, therefore that kind of thermal barrier in analysis was used.

\section{Formulation of the heat transfer problem}

The numerical simulations were made in the FreeFem ++ environment with the assumed flight duration of the shuttle of $t_{2}=2000 \mathrm{~s}$ and the heat flux [4]. Each axisymmetric model used in the research had 3 layers, i.e. aluminum structure of the vehicle, NOMEX SIP and the LI900 insulation. This simplification is due to the negligible effect of thin layers, Fig. 1.

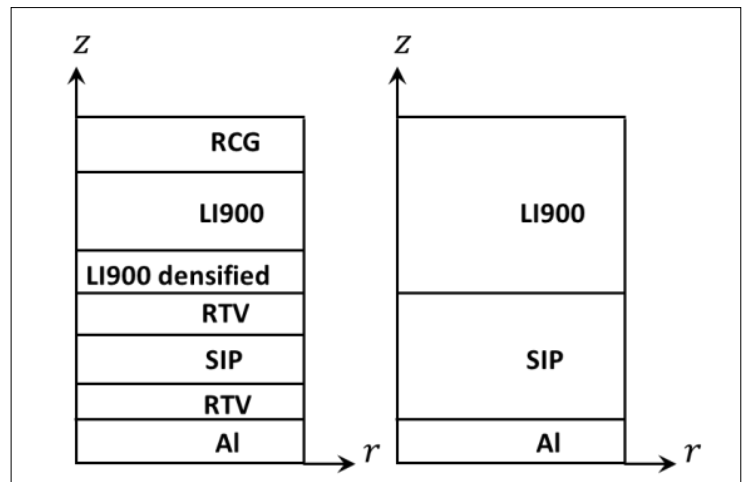

Fig. 1. Geometry of model.

The purpose of the analysis was to determine such a distribution of the concentration as to reduce the temperature increase on the surface of the aluminum structure. For this reason, the dopant was used at constant concentration throughout the entire insulation volume. The filings were oriented parallel to the outer surface of the insulation. In this way, in the insulation part of the heat flux was accumulated and conducted in the transverse direction of the main stream. Significant differences in maximum temperature should occur before 2000s when the orbiter is in flight.

In the investigations, a material loss of the diameter $\mathrm{D}$ of $1,2,3$ and $4 \mathrm{~cm}$ was assumed, Fig. 2. The additional conductive material, as a dopant, in the insulation was beryllium. The specifications of the HRSI tile material and additional material were taken from literature [5].

The first law of thermodynamics and Fourier's law was used in carrying out the analysis of the heat flow in non-stationary conditions for axisymmetric heat conduction model.

\footnotetext{
*Corresponding author: lukasz.brodzik@put.poznan.pl
} 


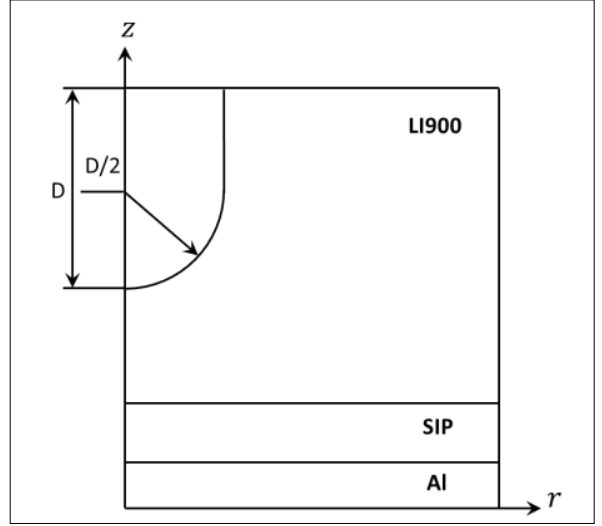

Fig. 2. Geometry of model.

Therefore, for the cylindrical coordinate system in space $\Omega_{1}$ the following formula was used (Fig. 3):

$$
\rho c_{p}\left(\frac{\partial T}{\partial t}\right)=\frac{1}{r} \frac{\partial}{\partial r}\left(k_{r} r \frac{\partial T}{\partial r}\right)+\frac{\partial}{\partial z}\left(k_{z} \frac{\partial T}{\partial z}\right)
$$

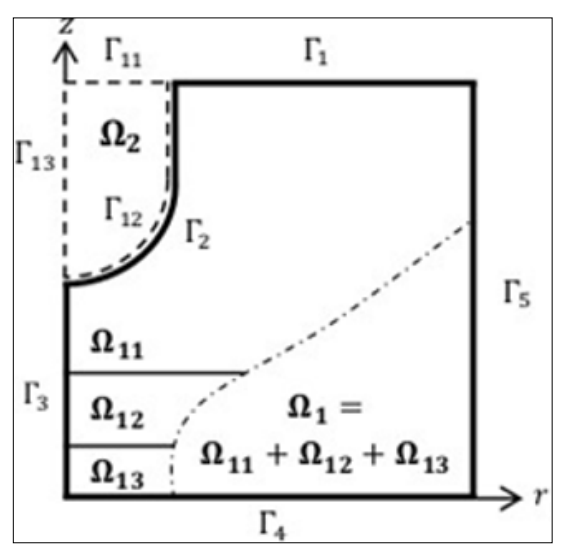

Fig. 3. Geometry of model.

In place of damage $\left(\Gamma_{12}\right.$ edge $)$ problem of radiation was solved using P1 approximation. This model assumes plane-parallel symmetry, that matter is in local thermodynamic equilibrium, scattering is coherent and isotropic. This model is one of approximations to solve Radiative Transfer Equation (RTE).

\section{Results}

For the smallest considered diameter $\mathrm{D}$, the addition of beryllium causes, that the maximum temperature on the surface of the underlying structure is less than the maximum working temperature of this material, Fig. 4.The increase of temperature in function of damage magnitude for $1 \%$ dopant of beryllium is described by the following polynomial

$$
T_{\max }=0.195 D^{2}-1.79 D+145.5
$$

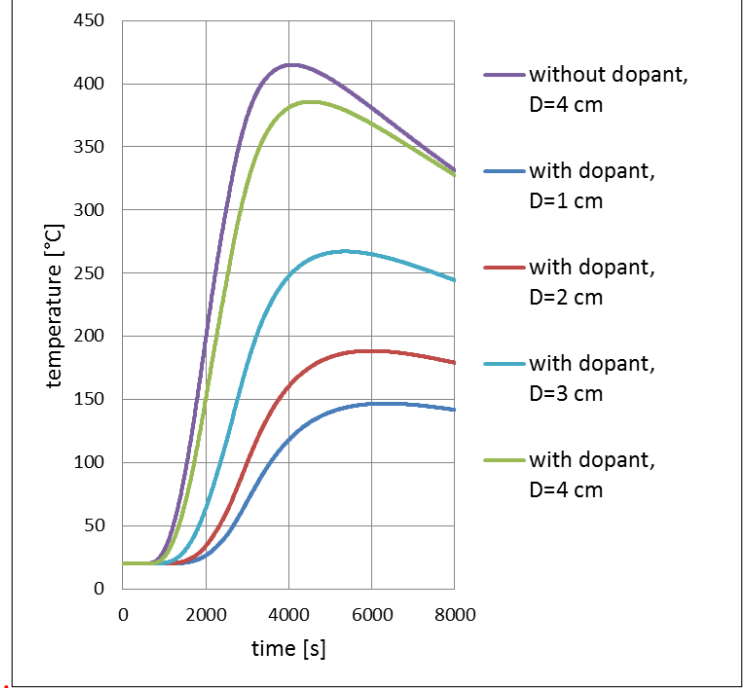

Fig. 4. Comparison of the distribution of temperature on the surface of the orbiter structure for a damaged tile, $\gamma=1 \%$.

Results are presented in Tab. 1.

Table 1. Results for models with $1 \%$ dopant of beryllium.

\begin{tabular}{|c|c|c|}
\hline $\mathbf{D}[\mathbf{c m}]$ & $\mathbf{T}_{\max }\left[{ }^{\circ} \mathbf{C}\right]$ & $\mathbf{t}[\mathbf{s}]$ \\
\hline 1 & 147 & 6305 \\
\hline 2 & 188 & 5975 \\
\hline 3 & 267 & 5350 \\
\hline 4 & 386 & 4525 \\
\hline
\end{tabular}

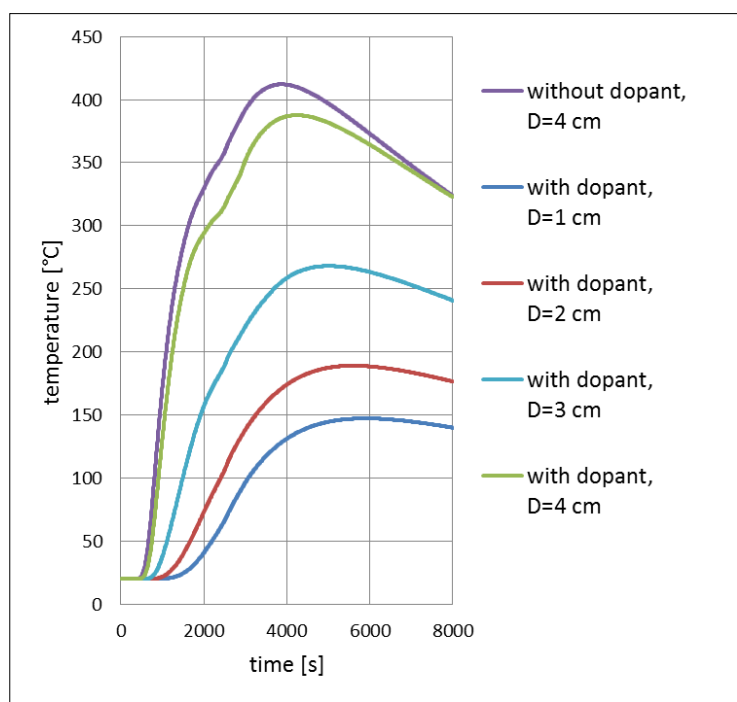

Fig. 5. Comparison of the distribution of temperature on the surface of SIP for a damaged tile, $\gamma=1 \%$.

Very similar distribution of temperature is observed on the surface of SIP, on merging with insulation, Fig. 5. 


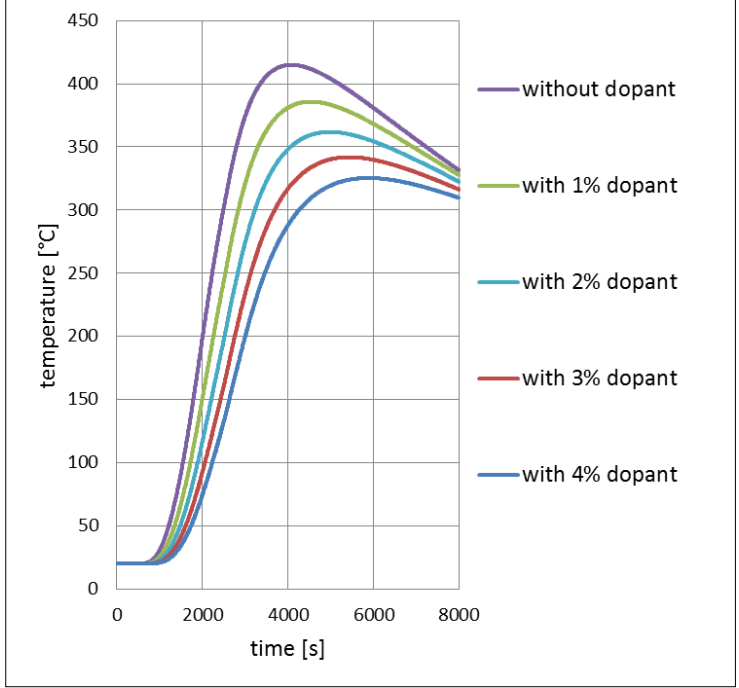

Fig. 6. Comparison of the distribution of temperature on the surface of the orbiter structure for a damaged tile, $\mathrm{D}=4 \mathrm{~cm}$.

The Fig. 6 shows, what is the effect of adding an increasingly large dopant of beryllium. The increase of temperature in function of dopant magnitude for $4 \mathrm{~cm}$ of damage diameter is described by the following polynomial

$$
T_{\max }=1.75 \gamma^{2}-29.05 \gamma+413.25
$$

Results are presented in Tab. 2.

Table 2. Results for models with different dopant of beryllium.

\begin{tabular}{|c|c|c|}
\hline$\gamma[\%]$ & $\mathbf{T}_{\max }\left[{ }^{\circ} \mathbf{C}\right]$ & $\mathbf{t}[\mathbf{s}]$ \\
\hline 1 & 386 & 4525 \\
\hline 2 & 362 & 4970 \\
\hline 3 & 342 & 5425 \\
\hline 4 & 325 & 5880 \\
\hline
\end{tabular}

It is possible to analyze how big delay in the occurrence of maximum temperature, as well as its reduction is provided by beryllium filings. There were compared for the same type of tile damage, $D=4 \mathrm{~cm}, 4$ variants of models. The first variant was a model, as before, with an insulation thickness of $77 \mathrm{~mm}$. The next two models contain insulation with dopant of beryllium equal to $1 \%$ of volume and insulation with an increased thickness equal to $86.868 \mathrm{~mm}$. Both insulations had the same weight of $0.2909 \mathrm{~kg}$. In the basic configuration, the insulation had a weight of $0.2579 \mathrm{~kg}$. The last option is a model in which dopant of so-called ideal material has been added. It had the properties of hydrogen characterized by a very high value of specific heat. The results are presented in the Fig. 7.

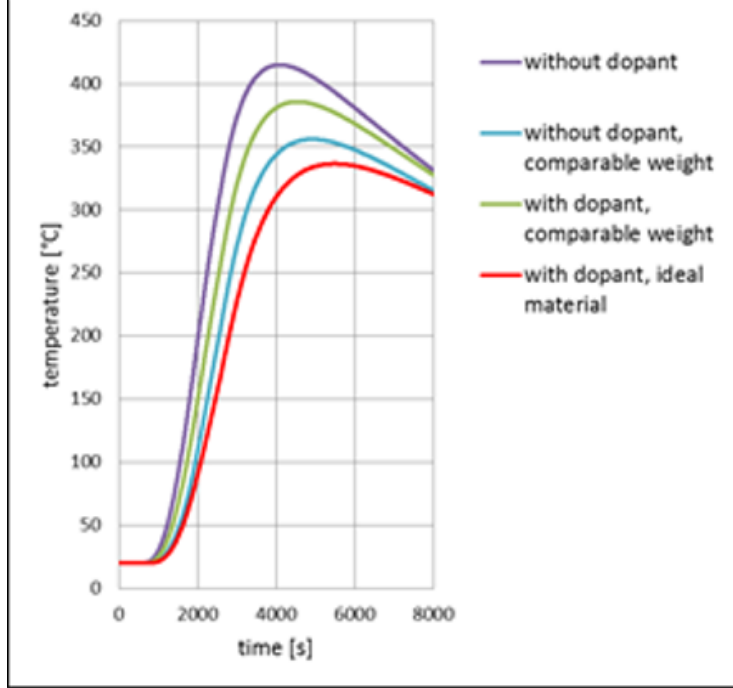

Fig. 7. Comparison of the distribution of temperature on the surface of the orbiter structure for a damaged tile, using the same weight, $\mathrm{D}=4 \mathrm{~cm}$.

Maximum temperature for model with thicker insulation is $357^{\circ} \mathrm{C}$ obtained after $4905 \mathrm{~s}$. For the corresponding model with a dopant of beryllium, these parameters are respectively $386^{\circ} \mathrm{C}$ and $4525 \mathrm{~s}$. The results related to the temperature distribution in the insulation including ideal material show, that using this method there can be delay the occurrence of its maximum additionally by almost 10 minutes.

\section{Conclusions}

The additional conductive layer should be made from a material characterized by a high value of specific heat and high melting point. These properties may turn out useful not only for future orbital flights but perhaps also for interplanetary flights. Such a material should also have a relatively high density. During the research, nickel was a good candidate in this respect ensuring good insulator properties. For the adopted distribution of the heat flux for the two considered additions of nickel during the flight, the structure did not exceed its admissible operating temperature range, hence was not damaged. Moreover, the insulation with an additional material should ensure limited heat conduction towards the orbiter structure. It should also be characterized by a greater conduction in the transverse direction (compared to the main direction of the heat flux).

It is important that the new material should be contained in an insulator in a low admixture value. This will provide a small increase in weight of the tile. Considered material, despite its small weight and relatively good thermal properties, proved to be useless in the proposed method. As a result, still thicker insulator layer exhibits more favorable heat transfer. The analysis proved, that the effect of the method used is similar to increasing the thickness of the insulator. However, there is the possibility of using such a material, which in practice proves to be more effective in reducing heat flow. Even ensuring a small reduction in 
maximum temperature in relation to the thicker insulation without an additional conductive layer but maintaining the same weight will bring big benefits. Using a modified material, the thickness of the insulator in the tile can be reduced. There may be tens of thousands of such tiles. Finally, it would reduce weight, and also cost of space missions.

\section{References}

1. Yuen W. W., Takara E., Cunnington G., Combined conductive/radiative heat transfer in high porosity fibrous insulation materials: theory and experiment, (The 6th ASME-JSME Thermal Engineering Joint Conference, 2003)

2 Balasubramaniam R., Callister S., Materials Science And Engineering: Indian Adaptation (Wiley India Pvt, 2009)

3 Dobrzański A. Leszek, Engineering materials and material design. Fundamentals of materials science and metallurgy, (WNT, 2006)

4. Ng Heok Wei, Thermomechanical analysis of a damaged thermal protection system, (doctoral thesis, University of Michigan, 2007)

5. Wiliams S. D., Curry M. Donald, Thermal Protection Materials-Thermophysical Property Data, (NASA Houston, 1992) 